\title{
Molecular Dynamics and Monte Carlo Simulations for Heat Transfer in Micro and Nano-channels
}

\author{
A.J.H. Frijns ${ }^{1}$, S.V. Nedea ${ }^{1}$, A.J. Markvoort ${ }^{2}$, A.A. van Steenhoven ${ }^{1}$, and \\ P.A.J. Hilbers ${ }^{2}$ \\ 1 Eindhoven University of Technology, Department of Mechanical Engineering, \\ P.O.Box 513, 5600MB Eindhoven, the Netherlands \\ 2 Eindhoven University of Technology, Department of Biomedical Engineering, \\ P.O.Box 513, 5600MB Eindhoven, the Netherlands
}

\begin{abstract}
There is a tendency to cool mechanical and electrical components by microchannels. When the channel size decreases, the continuum approach starts to fail and particle based methods should be used. In this paper, a dense gas in micro and nano-channels is modelled by molecular dynamics and Monte Carlo simulations. It is shown that in the limit situation both methods yield the same solution. Molecular dynamics is an accurate but computational expensive method. The Monte Carlo method is more efficient, but is less accurate near the boundaries. Therefore a new coupling algorithm for molecular dynamics and Monte Carlo is introduced in which the advantages of both methods are used.
\end{abstract}

\section{Introduction}

There is a tendency that mechanical and electrical components become smaller and smaller. Since most components produce heat when operating, it is essential to cool them in order to perform well and to ensure the life span of such components. For example in computer chips, the power increases with a factor of 10 every 6 years 9]. The standard cooling techniques start to fail. Therefore, more efficient cooling techniques, like microchannel cooling with phase transition, are necessary.

However when the channel size decreases, the continuum approach starts to fail. At Knudsen numbers $\gtrsim 0.1$, particle based methods should be used. From a physical point of view, molecular dynamics is a suitable method. From a computational point of view, this method is too expensive with respect to time to use for microchannels. To handle larger time frames, a Monte Carlo approximation is employed, but at the expense of less accuracy near the boundaries.

Our goal is to combine molecular dynamics with a Monte Carlo method such that we can use the advantages of both methods: molecular dynamics near the boundaries, because of the accuracy, and Monte Carlo in the bulk, because of the lower computational costs. 
In this paper, we consider both methods of modelling heat transfer in micro and nano-channels and introduce a new algorithm in which molecular dynamics and Monte Carlo codes with different time stepping are coupled.

\section{Molecular Dynamics and Monte Carlo Methods}

Molecular dynamics (MD) is a computer simulation technique where the time evolution of a set of interacting particles is followed. This is done by numerically solving the equations of motion (Newton's Second law) of classical multi-body systems. Given the positions, masses and velocities of all particles in the system and the forces on the particles, the motion of all (individual) particles can be followed in time by calculating the (deterministic) single particle trajectories. However, to calculate these trajectories is in general still very computationally intensive, because in principal it is possible that every particle interacts with every other particle in the system, resulting in $\mathrm{N}(\mathrm{N}-1) / 2$ pair interactions for a set of $\mathrm{N}$ particles that need to be calculated.

The interactions between the particles are given in the form of potentials. In order to model a gas by almost hard sphere interactions we use a truncated and shifted Lennard-Jones potential with a cut-off radius $R_{c}=2^{1 / 6} \sigma$. Where $\sigma$ is the collision diameter. For the simulations, we use the PumMa code developed at the Eindhoven University of Technology [4.

The Monte Carlo (MC) method is based on the Direct Simulation Monte Carlo method (DSMC) developed by Bird [3]. This DSMC method does not calculate the collisions exactly as in molecular dynamics, but generates collisions stochastically with scattering rates and post-collision velocity distributions determined from the kinetic theory of a dilute gas. Several authors [7/8|10] used this method successfully to study flow and heat transfer in microchannels for a dilute gas. However, for cooling purposes (high pressure or phase transition) we have also to model a dense gas in a microchannel. Therefore the DSMC method is modified by using the Enskog equation instead of the Boltzmann equation 5 6]. In this MC method the collisions take place between particles inside a cell and particles of neighbouring cells. The Enskog equation preserves the momentum and energy of the system.

In order to be able to couple molecular dynamics with Monte Carlo, both methods should give the same results for the limit situation where one artificial particle in the Monte Carlo simulation represents one molecule in the molecular dynamics simulation. As validation the particle density distribution and the temperature in a nanochannel are computed with both models. A nanochannel is chosen because of the computational costs. However, the method holds for microchannels too. We approximate the nanochannel by two infinite parallel plates kept at two constant temperatures $T_{c}=120 \mathrm{~K}$ and $T_{h}=240 \mathrm{~K}$. Gas molecules are confined between these walls. The interaction between the gas molecules and the walls is by thermal boundary conditions. A dense Argon gas is considered, with a particle diameter $R=0.191 \mathrm{~nm}$, the mean free path length $\lambda_{R}=0.346 \mathrm{~nm}$, and 
the initial uniform particle density $n_{0}=3.43$ particles $\mathrm{nm}^{-3}$. The total number of particles is $N=20000$.

The results for the MD and the MC codes are similar (fig. 11) and are in good agreement with Frezzotti's results [6]. The heat is transferred from the cold to the hot wall by the kinetic energy of the particles.

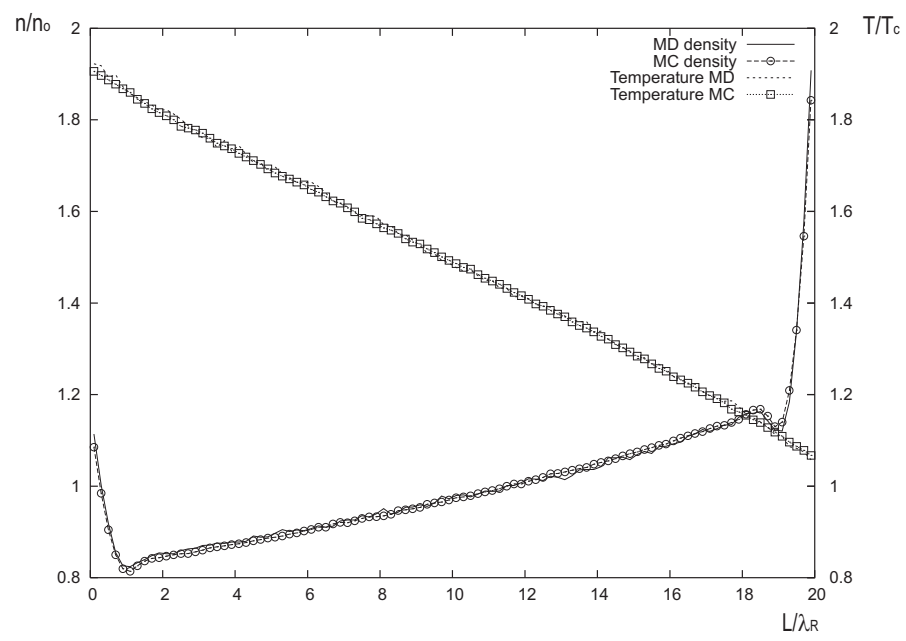

Fig. 1. Particle density profile $n / n_{0}$ and temperature profile $T / T_{c}$ in a nanochannel. Left: hot wall; right: cold wall

\section{Coupling of MD with MC}

As shown, both methods can be used to model the nanochannels. The MD method is accurate but computational expensive, while the MC method, in which an artificial particle represents several molecules, is more efficient. However, the boundary effects are computed less accurate, because the artificial particles are too large. The positions of the maxima and minima depend on the particle size. Therefore the MC method deviates from the MD method when several molecules are put in one MC particle. In order to get an accurate solution near the walls, a detailed model is needed in which the particle diameter is equal to the molecular diameter. MD is suited for that. In the bulk of the channel, the particle diameter is not so critical. In this region the computations can be sped up by MC with several molecules inside one artificial particle and by using larger time steps. So, a coupling of MD with MC results in a fast and accurate solution.

In our coupling algorithm, the domain is divided into $\mathrm{MD}$ and $\mathrm{MC}$ subdomains and an interface between these subdomains is defined (figure 2). The coupling algorithm is as follows. 
1. Assign particles to $M D$ or $M C$ part.

Particles are assigned to the MD or to the MC part depending on their position. All particles in regions I and II (see figure 2A) are assigned to the MD part and all particles in regions III and IV to the MC part. In the figure the particles that have been assigned to molecular dynamics are white whereas the MC particles are grey.

2. Perform $N$ Molecular Dynamics time steps.

Depending on the respective time step sizes in the MD and MC part of the simulation a certain number $(N)$ of MD steps corresponds with one single MC step. These $N$ MD steps are performed while keeping the positions of the MC particles fixed, as shown in figure $2 \mathrm{~B}$. The particles in region III are necessary because, although they are fixed in position, they can interact with the MD particles.

3. Send particles in range II and III from MD to $M C$.

The molecular dynamics time steps should be followed by a time step of the MC part. Before this can be done the particles in the interface regions II and III should be sent to the MC. As shown in figure $2 \mathrm{C}$, the particles are removed from the MD simulation and added to the $\mathrm{MC}$ simulation.

4. Perform one $M C$ time step.

One MC time step consists of two parts: an advection step and a collision step. The advection step, i.e. moving the particles, is done only for the MC (solid) particles, as shown in figure 2D. During the collision step collision pairs are randomly selected with the restriction that at least one of the particles should be a MC particle.

5. Send particles in range II and III from $M C$ to $M D$.

After the MC step the particles that are now in ranges II and III are sent to the MD simulation. Extra care should be taken here, because a MC particle can overlap with another particle, whereas this is impossible for particles in the molecular dynamics simulation. A solution to this problem is to check whether a MC particle in range II is too close to any MD particle or other $\mathrm{MC}$ particle in range II, and if so adding it a new random y and z position while keeping the $\mathrm{x}$ position fixed such that it no longer overlaps with any other particles.

6. Start over with step 1.

By converting the $\mathrm{MC}$ particles that ended up in range II to MD particles and vice versa MD particles that ended up in range III to MC particles (step 1) the next cycle of the simulation can be started.

Note that the MD and the MC steps are computed by different codes. They are coupled by an interface written in Python.

\section{Results and Discussion}

The algorithm is validated for the case in which the particles for the MC part have the same size as in the MD part. However, the time steps are different in both domains: one MC time step equals five MD time steps. We consider the 

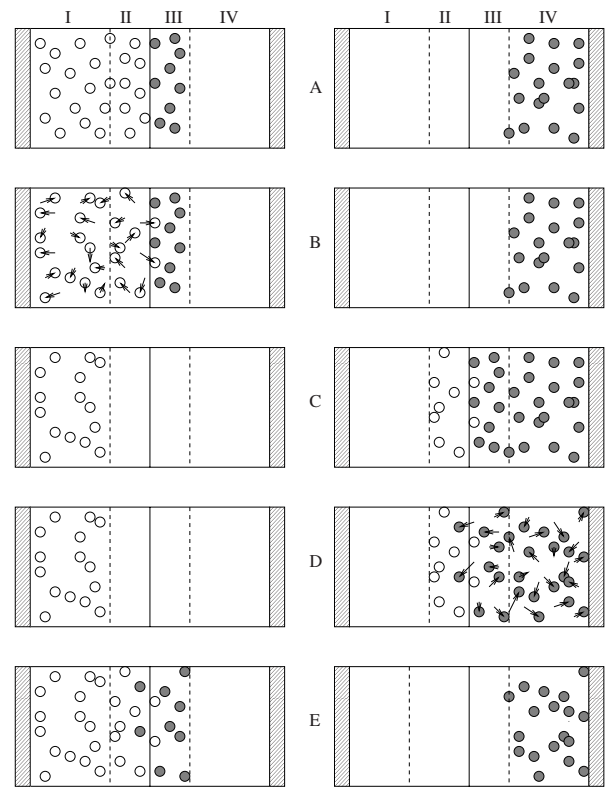

Fig. 2. Schematic view of the coupling algorithm. Left: MD steps; right: MC steps

nanochannel with the constant wall temperatures $T_{c}=T_{h}=120 \mathrm{~K}$. The other system parameters are chosen the same as in the first example. The results are plotted in figure 3 .

It is shown that the coupling of MD with $\mathrm{MC}$ by this interface is functioning. Some care must be taken at the artificial boundary between the $\mathrm{MC}$ and the MD domains, because the MC particles do not move while the MD steps are computed. In this way an artificial highly porous wall can be created. For dilute gases, this effect is negligible. However for a dense gas a small local disturbance in the particle density occurs.

\section{$5 \quad$ Future Research}

In this paper, it is shown that the algorithm for the coupling of $\mathrm{MD}$ with $\mathrm{MC}$ is functioning, but for a dense gas a small local disturbance in the particle density will occur at the interface. For the future, we will adapt the algorithm such that the local disturbance in the particle density will be minimised. To that end the interaction between the MD and MC particles in region III (step 2) will be improved by allowing small displacements of the MC particles. The next step will be that the particles in the MC domain consist of several MD particles. So, an extra step for combining several MD particles into one MC particle and disintegrating of one MC particle into several MD particles will be added to the algorithm. Finally, the efficiency of the algorithm will be studied in more detail. 


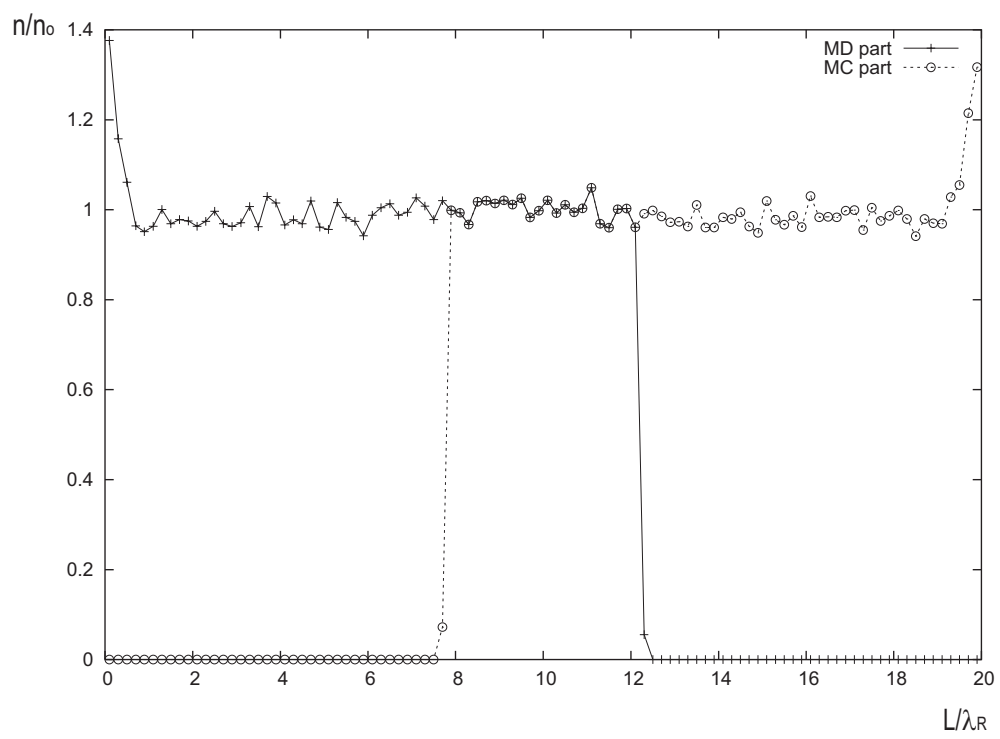

Fig. 3. Dimensionless particle density $n$ in a nanochannel with the coupling of the molecular dynamics method with the Monte Carlo method

\section{References}

1. Alexander, F.J., and Garcia, A.L., The Direct Simulation Monte Carlo method, Computers in Physics, 11 (1997), 588-593.

2. M.P. Allen and D.J. Tildesley, Computer Simulation of Liquids, Oxford University Press, 1987.

3. Bird, G.A., Molecular gas dynamics and the direct simulation of gas flows, Clarendon Press, Oxford, 1994.

4. K. Esselink, B. Smit, and P.A.J. Hilbers, Efficient Parallel Implementation of Molecular Dynamics on a Toroidal Network. Part I. Parallelizing Strategy, Journal of computational physics, 106 (1993), pp. 101-107.

5. Frezzotti, A., A particle scheme for the numerical solution of the Enskog equation, Phys. Fluids, 9 (1997), 1329-1335.

6. Frezzotti, A., Monte Carlo simulation of the heat flow in a dense sphere gas, European journal of mechanics, 18, (1999), 103-119.

7. Hadjiconstantinou, N.G. and Simek, O., Constant-Wall-Temperature Nusselt Number in Micro and Nano-Channels, Journal of Heat Transfer, 124 (2002), 356-364.

8. Mavriplis, C., and Ahn, J.C., and Goulard, R., Heat Transfer and Flowfileds in Short Microchannels Using Direct Simulation Monte Carlo, Journal of Thermophysics and Heat Transfer, 11 (1997), 489-496.

9. Schmidt, R.R., and Notohardjono, B.D., High end server low temperature cooling, IBM Journal of Research and Development, 46 (2002),739-751.

10. Yan, F., and Farouk, B., Computations of Low Pressure Fluid Flow and Heat Transfer in Ducts Using the Direct Simulation Monte Carlo Method, Journal of Heat Transfer, 124 (2002), 609-616. 